VIII ${ }^{\text {èmes }}$ Journées Nationales Génie Civil - Génie Côtier, Compiègne, 7-9 septembre 2004

\title{
Analyse des outils de calcul actuels dans le domaine maritime
}

\author{
Olivier Soulat
}

Ingénieur chargé d'études, CETMEF Compiègne

\section{Résumé:}

Les premiers retours d'expérience de ROSA 2000 montrent que la mauvaise adaptation des outils de calculs actuels au format de calcul semi-probabiliste complique la vérification aux états-limites des ouvrages. Une étude de recensement et d'évaluation des outils de calcul existant au niveau européen a été lancée. 154 logiciels ont pu être recensés. Leur étude a permis de constater que pour un ouvrage donné il existe un grand nombre de méthodes de calcul différentes, chaque pays privilégiant l'utilisation d'une ou plusieurs méthodes de reconnaissance géotechnique et de calcul. La proportion de logiciels adaptés à l'utilisation des coefficients partiels de sécurité reste faible (20\%). Afin de faciliter la mise en œuvre de ROSA 2000 des propositions sont faites pour adapter rapidement les logiciels existants.

\section{Abstract:}

First uses of ROSA 2000 showed that bad adaptation to semi-probabilistic format of present calculation tools complicates the limit-states verification of structures. A study made the inventory of the existing commercial softwares and evaluated their compatibility with the use of semi-probabilistic methods. 154 softwares were inventoried. The study of the softwares showed that for a type of structure there are different calculation methods, each country privileges one or several ground investigations and calculation methods. Only $20 \%$ of the softwares are adapted to the use of semi-probabilistic methods. In order to facilitate the use of ROSA 2000 several proposals are made for modernizing quickly the existing softwares.

Mots-clés : semi-probabiliste / eurocodes / logiciel / coefficient partiel / état-limite

\section{Introduction}

D’ici la fin de la décennie les Eurocodes deviendront d'application obligatoire dans l'ensemble des pays de l'Union Européenne (UE). Les Eurocodes utilisent le format de calcul semi-probabiliste avec la mise en œuvre de coefficients partiels de sécurité. En France comme dans les autres pays de l'UE, l'usage des Eurocodes va entraîner des changements par rapport aux méthodes de calcul traditionnelles qui utilisent des coefficients de sécurité globaux et qui sont utilisées pour de nombreux types d'ouvrages. Afin de préparer la transition entre les règlements actuels et les Eurocodes le CETMEF (Centre d'Etudes Techniques Maritimes et Fluviales) a rédigé puis diffusé les Recommandations pour le calcul aux états-limites des Ouvrages en Site Aquatique (ROSA 2000) ${ }^{(1)}$. Réalisé dans la seconde moitié des années 1990, ROSA 2000 se base sur les règles de calculs semi-probabilistes déjà existantes en France et dans les autres pays de l'UE ainsi que sur les versions d'application volontaire des Eurocodes (ENV).

ROSA 2000 a déjà été mis en œuvre sur au moins quatre projets. Les premiers retours d'expérience (écran mixte pieux et palplanches à Dunkerque ${ }^{(2)}$, quai sur pieux à Honfleur ${ }^{(3)}$ ) s’accordent sur 3 points : 
- à quelques exceptions près, les différents coefficients partiels ont été correctement calés par rapport aux méthodes anciennes, l'utilisation de ROSA 2000 n'entraîne par de surdimensionnement,

- de nombreux maîtres d'ouvrages et maître d'œuvre doivent faire l'objet d'une sensibilisation à la mise en œuvre des méthodes semi-probabilistes : commande d'une étude géotechnique pour calcul aux états-limites, réflexion approfondie sur l'utilisation de l'ouvrage, dialogue avec l'entrepreneur,

- la mise en œuvre des méthodes semi-probabilistes entraîne une accroissement sensible du nombre de calculs à réaliser. L'augmentation du volume des calculs entraîne un allongement de la durée (et donc du coût) des études d'autant plus important que la plupart des logiciels de calcul des ouvrages qui sont actuellement disponibles dans le commerce ne sont pas réellement adaptés à la mise en œuvre du format de calcul semi-probabiliste. En contre-partie, la rigueur nécessaire à l'analyse des cas de charge apporte une qualité supérieure aux ouvrages.

En partant de ce dernier constat le CETMEF a décidé de lancer une étude ${ }^{(4)}$ de recensement et d'évaluation du degré d'adaptation des logiciels actuellement utilisés pour le calcul des ouvrage portuaires.

\section{Les problèmes rencontrés avec les logiciels actuels}

L'introduction du format de calcul semi-probabiliste par ROSA 2000 n'entraîne pas de changement dans les méthodes de calcul existantes. L'utilisation des logiciels actuellement disponibles reste possible.

Le format semi-probabiliste nécessite de réaliser un certain nombre de combinaisons d'actions en appliquant des jeux de coefficients partiels sur l'ensemble des paramètres (résistances, caractéristiques de sols, actions variables et permanentes) afin de tenir compte de l'incertitude existante sur chacun d'eux ainsi que sur leur caractère favorable ou défavorable. La majorité des logiciels de calculs qui sont actuellement utilisés permet d'introduire seulement les valeurs de calculs (valeur caractéristique pondérée par un coefficient partiel). L’opérateur doit généralement calculer et introduire manuellement ces valeurs de calculs. En règle générale l'introduction des paramètres se fait au moyen de fichier de données que le logiciel peut lire. Il est nécessaire de créer autant de fichier de données qu'il y a de combinaisons (couramment plusieurs dizaines après simplifications).

La multiplication du nombre d'opérations manuelles est consommatrice de temps et potentiellement génératrice d'erreurs (qui sont d'autant moins décelables que le nombre de combinaisons est grand). Les premières expériences montrent qu'il est en général nécessaire de réaliser entre vingt et cinquante combinaisons différentes pour vérifier un ouvrage.

Il faut également remarquer qu'il n’existe pas non plus de système permettant de réaliser la synthèse des résultats.

\section{$\underline{\text { 3.Le recensement et l'évaluation du degré d'adaptation des logiciels }}$}

Le but de ROSA 2000 est de préparer le passage aux Eurocodes, le recensement des logiciels a été fait au niveau européen (pays membres du CEN à la fin de l'année 2003). Plus de 150 logiciels ont ainsi été recensés.

Les logiciels sont classés par type d'ouvrage en reprenant les titres des différentes parties de ROSA 2000 : murs de soutènement (calcul de la poussée des terres et de la capacité portante des fondations), gabions de palplanches, ouvrages sur pieux (quais et ducs d'Albe), écrans de soutènement, pentes et talus, parties en béton des ouvrages. Le recensement comprend également une partie sur les logiciels de calcul des tassements (la vérification à 
l'ELS nécessite le calcul des déplacements et des tassements) ainsi qu'une partie sur les logiciels de calcul aux éléments finis dont l'utilisation devrait connaître une forte croissance.

L'évaluation de l'adaptation des logiciels aux méthodes de calcul semi-probabilistes a été réalisée en utilisant les versions de démonstration des logiciels et en se basant sur les notices de ceux-ci (recherche des coefficients partiels à introduire dans les calculs, recherche des références à des règlements utilisant des coefficients partiels, études des formules de calcul données dans les manuels de référence...).

\section{$\underline{4 . C o n c l u s i o n s ~ s u r ~ l e ~ r e c e n s e m e n t ~}$}

\subsection{Absence de logiciels pour de nombreux états-limites}

La vérification de chaque ouvrage se fait en considérant un certain nombre d'états-limites. D'un état-limite à un autre, le nombre de logiciels du commerce disponible pour vérifier chacun varie considérablement. En règle générale pour les états-limites pour lesquels le recours au calcul informatique est indispensable (grand glissement, états-limites d'un écran de soutènement calculé par la méthode aux modules de réaction...) le nombre de logiciels disponible est généralement élevé (31 logiciels recensés pour le calcul des pieux de fondation), en revanche pour des états limites dont le calcul est relativement simple (stabilité au renversement, portance de fondations superficielles...) il n'existe aucun logiciel (ou un nombre très faible). Les états-limites simples à vérifier le sont en général au moyen de calcul manuels ou de logiciels (ou feuilles de calcul) établis au sein des entreprises et non diffusés dans le commerce.

\subsection{Grande diversité des méthodes de reconnaissances géotechniques et de calcul}

L'étude des manuels de référence et des interfaces d'introduction des données a permis de constater qu'il existe pour chaque ouvrage un grand nombre de méthodes de calcul. En général, les textes existants dans les différents pays conseillent ou imposent d'utiliser une méthode plutôt qu'une autre. Par exemple pour les calculs des écrans de soutènement ou des fondations, il existe des méthodes qui sont spécifiques à un pays ou un petit groupe de pays. Cette diversité des méthodes de calcul continuera à exister après la mise en application de l'Eurocode 7 qui n'impose pas de méthodes de calcul (les méthodes à utiliser seront fixées par les annexes nationales qui reprendront probablement celles des règlements nationaux existants). A titre d'exemple, la diversité des méthodes de calculs disponibles pour le calcul des soutènements est exposée dans un article ${ }^{(5)}$ qui décrit également les raisons qui ont amené à cette situation.

Au niveau des essais géotechniques (essais in-situ et en laboratoire), les différences entre pays sont encore plus marquées. Par exemple en France l'essai le plus courant est l'essai pressiométrique tandis qu'en Hollande, l'essai le plus courant est l'essai au pénétromètre statique. L’absence d'équivalence parfaite entre les résultats des différentes méthodes d'essai génère déjà des difficultés qui risque de croître nettement si plusieurs pays répondent à un appel d'offre en utilisant chacun leur méthode d'essai et de calcul.

\subsection{Nécessité de mettre à jour certaines méthodes de calcul}

Les premières utilisations de ROSA 2000 montrent que la réalisation de certaines vérifications peuvent poser problème (stabilité du massif d'ancrage...) car les formules sont utilisées à la limite ou en dehors de leur domaine de validité. On constate également que les méthodes actuellement utilisées pour calculer certains ouvrages (gabionnades, ouvrages en interaction avec d'autres ouvrages) ne sont pas satisfaisantes (hypothèses très simplificatrices, non prise en compte des interactions...). 
La mise en œuvre du format de calcul semi-probabiliste peut être une occasion de mettre à jour certaines méthodes de calcul ou bien de permettre la mise en œuvre de nouvelles méthodes.

\subsection{Adaptation des logiciels existants à l'utilisation des coefficients partiels}

L'étude a permis de constater que (dans le domaine géotechnique) seuls 30 logiciels (sur les 154 recensés) permettent de réaliser des calculs avec des coefficients partiels. On ne trouve des logiciels permettant de mettre en œuvre des coefficients partiels que lorsque la réglementation du pays où est vendu le logiciel impose l'utilisation de méthodes semiprobabilistes.

En revanche pour le calcul des structures en acier ou en béton la quasi-totalité des logiciels permettent l'utilisation de coefficients partiels des règlements existant ainsi que des versions provisoires des Eurocodes 2 et 3.

Les différences dans le degré d'adaptation aux méthodes semi-probabilistes peuvent s'expliquer par le fait que la plupart des règlements de calcul des structures en acier ou en béton font déjà référence au format semi-probabiliste tandis qu'une minorité de calcul géotechnique fait appel à ce format de calcul. Les logiciels permettant le calcul semiprobabiliste se développeront probablement après la publication des annexes nationales dans les différents pays.

\subsection{Le cas des logiciels de calcul aux éléments finis}

\subsubsection{Diversité des logiciels disponibles}

Vingt-six logiciels de calcul aux éléments finis ont été recensé. Certains logiciels permettent de traiter un type d'ouvrages précis (par exemple la stabilité d'un talus). On trouve également des logiciels d'usage général qui permettent de traiter l'ensemble des problèmes de géotechnique. Certain d'entre eux ont été développés spécifiquement pour le calcul géotechnique, ils ont fait l'objet de vérification et de comparaison par rapport à des ouvrages réels ou des modèles réduits. Ces travaux ont permis de caler les différents paramètres, de tester des modèles de sol et de les fiabiliser. Parmi les logiciels recensés cinq n’étaient initialement pas destinés au calcul géotechnique : la majorité d'entre eux n’a pas fait l'objet de publication sur leurs vérifications en calcul géotechnique il y a donc lieu de les utiliser avec précautions.

\subsubsection{Evolutions futures}

Les logiciels de calculs aux éléments finis permettent de traiter des problèmes couplés (par exemple écran de soutènement avec une nappe variant sous l'effet des marées), donnant ainsi une modélisation plus fine du comportement de l'ouvrage. Leur utilisation est facilitée par des interfaces conviviales.

Le calcul aux éléments finis est susceptible de remplacer les méthodes de calculs qui ont besoin d'être mises à jour (gabionnades, stabilité du massif d'ancrage, interactions diverses entre les ouvrages...)

Leur utilisation nécessite un certain nombre de précautions: emploi d’une personne formée à l'utilisation du logiciel, mise en place de nouvelles méthodes d'essais destinées à évaluer les nouveaux paramètres à introduire dans certains modèles de sol (problème déjà rencontré avec le calcul aux modules de réaction).

Les difficultés d'établissement des paramètres de sol sont illustrées par un article sur un concours de prédiction de comportement d'un ouvrage instrumenté( ${ }^{(6)}$ : à partir des mêmes résultats d'essais, les différentes équipes ont déterminé des valeurs très différentes pour certains paramètres ce qui a abouti à des écarts considérables lors de la comparaison des 
résultats : déplacement calculés variant de 0 à $60 \mathrm{~mm}$, moments calculés variant d'un facteur 4.

Aucun des logiciels de calculs aux éléments finis qui ont été recensés ne permet d'utiliser des coefficients partiels. Des travaux destinés à déterminer la façon dont les coefficients partiels doivent être utilisés dans le cadre d'un calcul aux éléments finis sont actuellement en cours $^{(7)}$.

\section{Quelques propositions d'adaptation des logiciels}

Dans l'attente de la sortie des logiciels de calcul adaptés à l'utilisation du format de calcul semi-probabiliste des solutions simples sont envisagées afin d'encourager les services à mettre en œuvre ROSA 2000.

La création de logiciels adapté à l'usage des coefficients partiels ne doit pas faire oublier qu'il est impératif de réduire le nombre de combinaisons à vérifier à un nombre acceptable (moins de 50 dans les projets déjà réalisés) grâce à une réflexion préalable sur le comportement de la structure et sur son usage.

\subsection{L'existant}

\subsubsection{Les logiciels de calcul automatique}

Au cours des dernières années des logiciels utilisant des tableurs ont été développés. La SOGREAH (calcul des quais poids) et le CETMEF (calcul des ducs d'Albe souples) ont créé des feuilles de calcul. Ces outils permettent de réaliser automatiquement l'ensemble des combinaisons de coefficients partiels.

Ces méthodes de calculs présentent cependant un certain nombre d'inconvénients. Le nombre de paramètres utilisés dans les calculs doit rester suffisamment faible (pour $\mathrm{n}$ paramètres avec 2 coefficients partiels on a généralement $2^{n}$ combinaisons à vérifier). Dans le cas du logiciel de calcul des murs poids les utilisateurs aboutissent, après simplification, au calcul de plus de mille combinaisons. La lecture des résultats est en général difficile et il est nécessaire de vérifier que les combinaisons dimensionnantes sont possibles physiquement. D'autre part un des buts de ROSA 2000 est d'amener les différents acteurs à s'interroger sur l'usage et le fonctionnement de l'ouvrage, le fait de réaliser automatiquement les combinaisons peut amener à omettre ou à réduire cette phase très importante.

Pour un ouvrage simple et un nombre réduit de variable le nombre de calculs à effectuer est élevé (16 calculs pour chaque catégorie d'état-limite dans le cas du calcul de Duc d'Albe). L'utilisation de ce type de logiciels est restreinte aux méthodes de calculs simples et d'exécution rapide. Avec un nombre de combinaisons élevé l'utilisation de méthodes de calcul lente aboutit rapidement à des temps de calcul inacceptables.

\subsubsection{Les logiciels se basant sur les règlements existants}

Dans les différents pays de l'Union Européenne, il existe déjà des règlements utilisant le format de calcul semi-probabiliste. En France c'est notamment le cas du calcul de fondation (fascicule 62 titre $\mathrm{V}$ du CCTP) et du béton armé ou précontraint (fascicule 62 titre I ou BAEL-BPEL). Ces règlements existant depuis plus d'une dizaine d'années et étant d'application obligatoire, des logiciels tenant compte du format de calcul semi-probabiliste sont déjà disponibles sur le commerce. C'est notamment le cas des logiciels de calcul des structures en béton armé qui sont conçus pour être utilisés avec des règlements semiprobabilistes de plusieurs pays voir les Eurocodes. Dans ces logiciels les valeurs des différents coefficients partiels rentrées par défaut sont celles des règlements nationaux existant, les utilisateurs peuvent cependant modifier les valeurs de ces coefficient partiel notamment s’ils utilisent des règlements préconisant des valeurs différentes. 
Ces logiciels adaptés à la mise en œuvre des méthodes semi-probabilistes montrent également les différentes solutions qui ont été adoptées pour permettre à l'utilisateur l'introduction des coefficients partiels (tableaux, écriture des combinaisons...) dont les personne chargé de mettre à jour les logiciels pourront s’inspirer.

\subsection{Pistes à explorer}

\subsubsection{Les interfaces}

Dans le passé le CETMEF a développé et a diffusé aux services du ministère de l'équipement un grand nombre de logiciels qui sont encore utilisés de nos jours. Tous ces logiciels fonctionnent encore sous DOS ce qui les rend peu conviviaux (introduction des données sans souris, impossibilité de visualiser l'ouvrage...) et difficiles à utiliser (ces logiciels ne sont pas compatibles avec les systèmes d'exploitations actuels : problème d'affichage, d'impression...).

Depuis quelques années le CETMEF a lancé une opération de modernisation de ces logiciels en créant des interfaces qui permettent à l'utilisateur de saisir plus facilement les données et de faciliter l'exploitation des résultats.

Tous ces logiciels sont capables de lire des fichiers de commande (fichiers textes pouvant être édités avec des traitements de texte par exemple). Ces fichiers permettent seulement d'utiliser les valeurs de calcul des paramètres. Actuellement les programmes servant d'interface permettent de traduire les données rentrées par l'utilisateur en un ou plusieurs fichiers. On peut envisager d'entrer les combinaisons de coefficients partiels que l'on souhaite utiliser, le programme effectue les opérations de pondération et crée autant de fichiers de données (contenant les valeurs de calcul des différents paramètres) qu'il y a de combinaisons.

\subsubsection{Les tableurs}

La plupart des logiciels anciens utilisent des fichiers de données avec une syntaxe simple : une seule colonne de valeur avec éventuellement des mots clefs servant d'instructions.

La réalisation d'une interface nécessite le travail d'un spécialiste et peut s'avérer rapidement coûteuse ; Pour palier ce problème on peut également imaginer d'utiliser des tableurs pour créer facilement les fichiers de données à partir des valeurs caractéristiques des différents paramètres et des jeux de coefficients à appliquer. Cette solution présente l'avantage d'être simple à mettre en œuvre. Chaque bureau d'études peut développer son propre outil comme c'est déjà le cas pour le calcul des états-limites simples à vérifier (voir 0). En dépit de leur simplicité ces outils devront faire l'objet de tests de validation.

Dans ce cas l'utilisateur doit lancer lui-même les différents calculs, il est également nécessaire qu'il réalise lui-même la synthèse des résultats obtenus pour les différentes combinaisons.

\section{Evolutions entre ROSA 2000 et les Eurocodes}

Depuis la rédaction de ROSA 2000 les Eurocodes ont évolué. Aujourd'hui un certain nombre d'Eurocodes sont sur le point d'être votés ou l'ont déjà été. L’étude a montré qu'il existe des différences entre ROSA 2000 et les Eurocodes achevés (par exemple dans l'Eurocode 7 modification de la pondération liée au remplacement des cas A, B et C par les approches de calcul 1,2 et 3).

On observe que les valeurs de certains coefficients partiels ont évolué (certaines de ces valeurs données dans les Eurocodes sont indicatives il faudra attendre la parution des annexes nationales pour connaître leurs valeurs définitives). Il est nécessaire que les logiciels ayant 
subi une adaptation dans le cadre de la mise en œuvre de ROSA 2000 laissent à l'utilisateur la possibilité de modifier manuellement les coefficients partiels.

On observe également des évolutions sur les méthodes de calcul et les méthodes d'introduction des coefficients partiels. C'est par exemple le cas du calcul des structures en béton (introduction par l'Eurocode 2 de bielles et de tirants d'inclinaison variable pour les vérifications à l'effort tranchant, calcul de l'ouverture des fissures pour les calculs ELS, changement de méthode pour tenir compte de la durabilité de l’ouvrage et de l'agressivité du milieu).

Ces changements imposent la réalisation de nouveaux logiciels capables de faire les vérifications avec les anciennes méthodes (pour la période de coexistence des anciens règlements et des Eurocodes) et les nouvelles méthodes. Il sera nécessaire d'apporter des modifications à ROSA 2000 après la publication de toutes les versions définitives des Eurocodes.

\section{$\underline{\text { 7.Conclusion }}$}

L'étude a montré qu'il existe dans l'Union Européenne un grand nombre de logiciels permettant le calcul des ouvrages portuaires. Les différences existantes entre les méthodes de calcul des différents pays expliquent en partie le nombre de logiciels disponibles dans le commerce. Le faible proportion (20\%) de logiciels permettant l'utilisation de coefficients partiels est probablement lié au fait qu'il existe encore très peu de règlements imposant l'utilisation du format semi-probabiliste pour les calculs géotechniques. On peut penser que les logiciels adaptés à l'utilisation des coefficients partiels apparaîtront sur le marché à partir de la date de publication de l'Eurocode 7 et des différentes annexes nationales.

En attendant la publication de logiciels adaptés il existe un certain nombre de solutions simples et peu coûteuses qui permettent d'adapter les logiciels existant pour faciliter la tâche des personnes qui souhaitent se former à l'utilisation des méthodes semi-probabilistes pour la vérification des ouvrages portuaires.

\section{8.références}

1.ROSA 2000 (2001), Recommandations pour le calcul aux états-limites des Ouvrages en Site Aquatique, CD-ROM, CETMEF CSTB, Ministère de l'équipement des Transports et du Logement

2.SOULAT O. (2002) «Application de ROSA 2000 à un projet de rideau de soutènement mixte », rapport de stage, CETMEF Ecole Nationale des Travaux Publics de l'Etat

3.BAGUELIN F. SIGROS B. DERRIEN P. KOVARIK JB. LEGROS L. (2003) "Application des Eurocodes au dimensionnement d'un quai sur pieux: le retour d’expérience », article rédigé pour une réunion tenue à Paris

4.SOULAT O. (2003) «Outils de vérification des ouvrages portuaires et leur adaptation aux méthodes semi-probabilistes », rapport, CETMEF Ecole Nationale des Ponts et Chaussées

5.DELATTRE L. (2001) «Un siècle de méthodes de calcul d'écrans de soutènement. I l'approche par les méthodes classiques et la méthode aux coefficients de réaction » Bulletin des laboratoires des ponts et chaussées, $\mathrm{n}^{\circ} 234$, pages 36 à 55

6.MESTAT P. et ARAFATI N. (1998) «Modélisation par éléments finis du comportement du rideau de palplanches expérimental de Hochstetten » Bulletin des laboratoires des ponts et chaussées, $\mathrm{n}^{\circ} 216$, pages 19 à 39

7.BAUDUIN C. DE VOS M. SIMPSON B. (2000) « Some considerations on the use of finite element methods in ultimate limit state design » LSD 2000, international workshop on limit state design in geotechnical engineering, Melbourne, Autsralie 